\title{
Modelado de Maxent, predicción de la distribución espacial de la vicuña en Ecuador
}

\author{
DOI: https://doi.org/10.33262/ap.v3i3.1.109
}

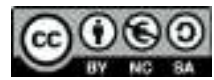

\section{Modelado de Maxent, predicción de la distribución espacial de la vicuña en Ecuador}

\author{
Maritza Lucía Vaca Cárdenas. ${ }^{1}$, Byron Ernesto Vaca Barahona. ${ }^{2}$, Diego Francisco \\ Cushquicullma Colcha. ${ }^{3}$ \& Guicela Margoth Ati Cutiupala. ${ }^{4}$
}

\begin{abstract}
.
Introduction. In 1988 South American camelids were reintroduced in the Chimborazo Fauna Production Reserve, the emblematic species being the vicugna vicugna. Objective. Determine the current and potential spatial distribution of the vicuña and evaluate the contribution of the variables to establish the suitability of the habitat. Methodology. The research was non-experimental, field, evaluative level and proposes environmental, topographic and anthropic guidelines and criteria for a sustainable management of the species. At first the presences of the individuals were geo-referenced, then the layers of the variables were prepared: NDVI, NDWI, DEM, precipitation, land use, temperature and type of ecosystems and finally the Maxent software model was run and evaluated contributions. Results. $63.7 \%$ of the RPFCH has sites that are not suitable for vicuña, $26.1 \%$ are considered potential distribution areas, $10.1 \%$ occupy moderately suitable habitat and finally only $0.1 \%$ corresponds to highly suitable habitat. adequate since it meets the favorable conditions for the survival and reproduction of the species. Conclusions. The variable that most contributes to establishing the suitability of the

${ }^{1}$ Escuela Superior Politécnica de Chimborazo, Facultad de Ciencias Pecuarias. Riobamba, Ecuador. maritza.vaca@espoch.edu.ec, ORCID 0000-0003-4474-4354

${ }^{2}$ Escuela Superior Politécnica de Chimborazo, Facultad de Ingeniería Electrónica. Riobamba, Ecuador. byron.vaca@espoch.edu.ec, ORCID 0000-0002-3622-0668

${ }^{3}$ Escuela Superior Politécnica de Chimborazo, Facultad de Recursos Naturales. Riobamba, Ecuador. diego.cushquiculma@espoch.edu.ec, ORCID 0000-0001-6265-8164

${ }^{4}$ Universidad de Granada, Facultad de Ciencias. Granada, España. guicelaati@correo.ugr.es, ORCID 0000-0002-9779-2758
\end{abstract}


habitat is the NDVI, this detects areas with low biomass density, but with nutritional quality of the forage. $63.7 \%$ of the RPFCh are sites that are not suitable for the species, while only $36.3 \%$ correspond to highly suitable, moderately suitable and moderately low areas, which calls for the establishment of medium and long-term measures for the management of the species.

Keywords: Spatial distribution, habitat, suitability, predictive capacity, vicuña, maxent

\section{Resumen.}

Introducción. En 1988 se reintrodujeron camélidos sudamericanos en la Reserva de Producción de Fauna Chimborazo, siendo la especie emblemática la vicugna vicugna. Objetivo. Determinar la distribución espacial actual y potencial de la vicuña y evaluar la contribución de las variables para establecer la idoneidad del hábitat. Metodología. La investigación fue no experimental, de campo, de nivel evaluativo y propone lineamientos y criterios ambientales, topográficos y antrópicas para un manejo sostenible de la especie. En un primer momento se geo referenció presencias de los individuos, luego se prepararon las capas de las variables: NDVI, NDWI, MDE, precipitación, uso del suelo, temperatura y tipo de ecosistemas y finalmente se corrió el modelo del software Maxent y se evaluaron las contribuciones. Resultados. El $63,7 \% \mathrm{RPFCH}$ posee sitios no adecuados para la vicuña, el $26,1 \%$ se considera como zonas potenciales de distribución, el hábitat moderadamente adecuado lo ocupa el $10,1 \%$ y finalmente apenas el $0,1 \%$ corresponde al hábitat altamente adecuado puesto que reúne las condiciones favorables para la supervivencia y reproducción de la especie. Conclusiones. La variable que más contribuye a establecer la idoneidad del hábitat es el NDVI, este detecta zonas con densidad de biomasa baja, pero con calidad nutricional del forraje. El 63,7\% de la RPFCh son sitios no adecuados para la especie, mientras apenas el 36,3\% corresponden a zonas altamente adecuadas, moderadamente adecuado y moderadamente bajo, que insta a plantear medidas a mediano y largo plazo para el manejo de la especie.

Palabras claves: distribución espacial, hábitat, idoneidad, capacidad predictiva, vicuña, maxent.

\section{Introducción}

El proceso de reintroducción de vicuñas inicia con la suscripción del convenio para la conservación y manejo de la vicuña en la ciudad de Lima el 20 de Diciembre de 1979 entre Argentina, Bolivia, Chile, Ecuador y Perú(Ministerio del Ambiente del Ecuador, 2002). En 1987 se crea la Reserva de Producción de Fauna Chimborazo con Registro Oficial No. 806 del 9 de noviembre de 1987 con el fin de fomentar la crianza de las diferentes especies de camélidos sudamericanos y, en 1988 se liberaron 200 vicuñas (100 machos y 100 hembras) de Chile y Perú, en 1993 se realizaron donaciones adicionales que alcanzaron un total de 373 vicuñas (Albán-Guaman, 2009), que se han crecido poblacionalmente llegando a reportarse en año 2016, 7021 individuos (Rodríguez González \& Morales De La Nuez, 2017), que habitan en los páramos del área protegida. 
La zona también alberga 42 comunidades indígenas que efectúan actividades agrícolas y pecuarias las cuales han generado un crecimiento sustancial de las áreas cultivables incluso sobre los $4100 \mathrm{msnm}$, mermando el hábitat de la vicuña lo que genera un conflicto gente fauna. El presente estudio plantea determinar la distribución espacial actual y potencial de la vicuña y evaluar las contribuciones de las diferentes variables, los resultados apoyarán la gestión de actores, tomadores de decisiones para la implementación de medidas de conservación y manejo sostenible de vicugna vicugna.

\section{Marco teórico}

A lo largo de los años estudiosos han demostrado la importancia de las zonas altoandinas para la humanidad (Hofstede, 2004; Rodríguez González \& Morales De La Nuez, 2017; Vásconez et al., 2001), pues la conservación y manejo de este entorno natural son claves para el bienestar y desarrollo humano (Varea, 2004), no obstante, es innegable la afectación que sufren estas debido a actividades antropogénicas como sobrepastoreo, prácticas de manejo inadecuadas y por efectos del cambio climático (Herrador-Valencia $\&$ Paredes, 2016; Podwojewski Pascal et al., 2002). Estos problemas no distan de lo que ocurre en las áreas protegidas pues a pesar de ser declaradas áreas dedicadas a la conservación esta premisa no siempre se cumple, una de las reservas de protección más importantes del Ecuador es la Reserva de Producción de Fauna Chimborazo (RPFCH) ubicada en la zona central del Ecuador en las provincias de Chimborazo, Bolívar y Tungurahua.

La RPFCH fue creada bajo Acuerdo Ministerial No. 437 del 26 de octubre de 1987 publicado en el Registro Oficial No. 806 del 9 de noviembre del mismo año(Ministerio del Ambiente del Ecuador, 2002), el objetivo fundamental de su declaratoria consistía en apoyar el desarrollo sostenible de las 41 comunidades que habitan en los límites de la $\mathrm{RPFCH}$, además de ensayar la reintroducción de camélidos, protección de los páramos y apoyar a la investigación científica. Una de las especies que habita el AP es la vicuña (Vicugna vicugna) (Tirira, 2015), un camélido silvestre que comparte hábitat con los otros camélidos del altiplano (González, 2020), característicos de Sudamérica (Torres, 1983). Estás especies tienen un rol clave en los ecosistemas altoandinos, además de un alto valor cultural en la cosmovisión de los pueblos andinos (Rojo \& Yanira, 2012), a esto se suma el valor económico pues la fibra de vicuña posee un valor elevado en el mercado (GarcíaHuamaní, 2020), y sin embargo se encuentra seriamente amenazada.

En Ecuador la vicuña está adaptada principalmente al arenal (Rodríguez González \& Morales De La Nuez, 2017) uno de los ecosistemas que conforman la RPFCH, sus características morfológicas evitan la degradación de los suelos y permite la regeneración de los pastos de los que se alimenta, a diferencia del ganado doméstico introducido (Korswagen Eguren, 2016), pues la reserva de producción de fauna Chimborazo, alberga a 42 comunidades indígenas (Ati-Cutiupala et al., 2021) que efectúan actividades socioeconómicas, evidentemente existe una superposición entre el uso de espacios dedicados a las actividades comunales y el hábitat de las vicuñas silvestres, esto genera 
un dilema entre conservar el hábitat de la vicuña o el aprovechamiento económico por parte de las comunidades.

Bajo este argumento es necesario estudiar los patrones de distribución y para esto se han desarrollado los modelos de distribución de especies (MDE) que son herramientas empleadas para estudiar patrones espaciales en ecología, contribuyen a los procesos de conservación y planificación (Hernández et al., 2018; Korswagen Eguren, 2016), buscan clarificar la utilidad y el potencial que tienen los modelos de distribución, con el objetivo de invitar a la investigación en biogeografía y así seguir avanzando en el conocimiento de los patrones espaciales y temporales de la distribución de los organismos(Pliscoff \& Fuentes-Castillo, 2011).

El modelo de entropía máxima (Maxent) puede usar variables ambientales, físicas y puntos de presencia de especies para calcular las restricciones y explora la posible distribución de la entropía máxima bajo esta condición de restricción, y luego predice la idoneidad del hábitat de las especies en el área de estudio (Merow et al., 2013; Phillips et al., 2006), para el presente estudio se incorporan tres tipos de información: ocurrencias de las especies, variables ambientales y factor antrópico

En este contexto la investigación plantea el análisis de las relaciones espaciales entre el uso del suelo y el hábitat de la vicuña, con la finalidad de determinar el efecto de las actividades socioeconómicas en la dinámica del hábitat de la especie. Para ello se determinaron, la distribución actual y el hábitat potencial de la vicuña.

\section{Área de estudio}

La zona de estudio comprende el territorio de la reserva de producción de fauna Chimborazo, la cual está situada en la provincia del mismo nombre, además de ocupar gran parte de Tungurahua y parte de la provincia de Bolívar (Figura 1). Sus coordenadas son 743536 E 9838888 S en metros, posee una extensión de 52560 hectáreas distribuidas en altitudes que van desde los 3800 hasta los 6268 metros sobre el nivel del mar (msnm) (Jara et al., 2019; MAE, 2014).

Las variaciones de temperatura en la RPFCH ocurren desde $-4,80{ }^{\circ} \mathrm{C}$ registrada como temperatura mínima y ocurre en el mes de diciembre, la temperatura máxima alcanza los $11,40^{\circ} \mathrm{C}$ durante el mes de noviembre(Vistin Guamantaqui et al., 2020), debido a la altitud es frecuente que en los días más fríos y húmedos la precipitación se presente en forma de nieve o escarcha en las zonas más altas(Ati-Cutiupala et al., 2021), el área registra un promedio anual de $998 \mathrm{~mm}$ que varía entre $809 \mathrm{~mm}$ en las zonas menos pluviosas y los $1300 \mathrm{~mm}$ en las zonas de mayor húmedad (Ministerio del Ambiente del Ecuador, 2014).

En la reserva de producción de fauna Chimborazo se presentan amenazas para la conservación, siendo la agricultura y ganadería una de las actividades económicas más importantes y recurrentes en la zona (Ati-Cutiupala et al., 2021), generando gran presión 
sobre el área de conservación (Hofstede et al., 2015; Romero et al., 2018) y las especies que allí habitan.

En 2013 el Ministerio de Ambiente Agua y Transición Ecológica (MAATE) estableció una clasificación ecosistémica para Ecuador, la RPFCH alberca ocho tipos de ecosistemas; Herbazal ultra húmedo subnival del Páramo(4400-4900 msnm), Herbazal húmedo montano alto superior del Páramo (3500-4200 msnm), herbazal húmedo subnival del Páramo ( 3400-4300), Herbazal y Arbustal siempreverde subnival del Páramo (41004500 msnm), Herbazal del Páramo (3400-4300), Arbustal siempreverde y Herbazal del Páramo (3300-3900), esta clasificación ecosistémica incluye páramo herbáceo y páramo de almohadillas, además de gelidofitia (Sierra, 1999), por otro lado de acuerdo a Josse et al (2003) la clasificación propuesta por MAATE incluye a pajonales altimontanos y montanos paramunos, pajonales arbustivos altimontanos paramunos y pajonal edafoxerofilo altimontano paramuno, vegetación geliturbada y edafoxerofila subnival paramuna (Josse et al., 2003). En esencia este mosaico de formaciones vegetales se resume un paisaje de alta montaña con presencia de nevados, lagunas de origen glaciar y riachuelos, laderas empinadas, afloramientos rocosos, colinas y una amplia cobertura de pastizales. Los pastizales se clasifican en pajonales y bofedales, los cuales se diferencian por la composición de especies vegetales (Korswagen Eguren, 2016).

Gran parte de los pastizales son empleados para el pastoreo por las comunidades, ya que en el territorio gran parte de los suelos no son aptos para la agricultura.

\section{Figura 1}

Localización geográfica del área de estudio

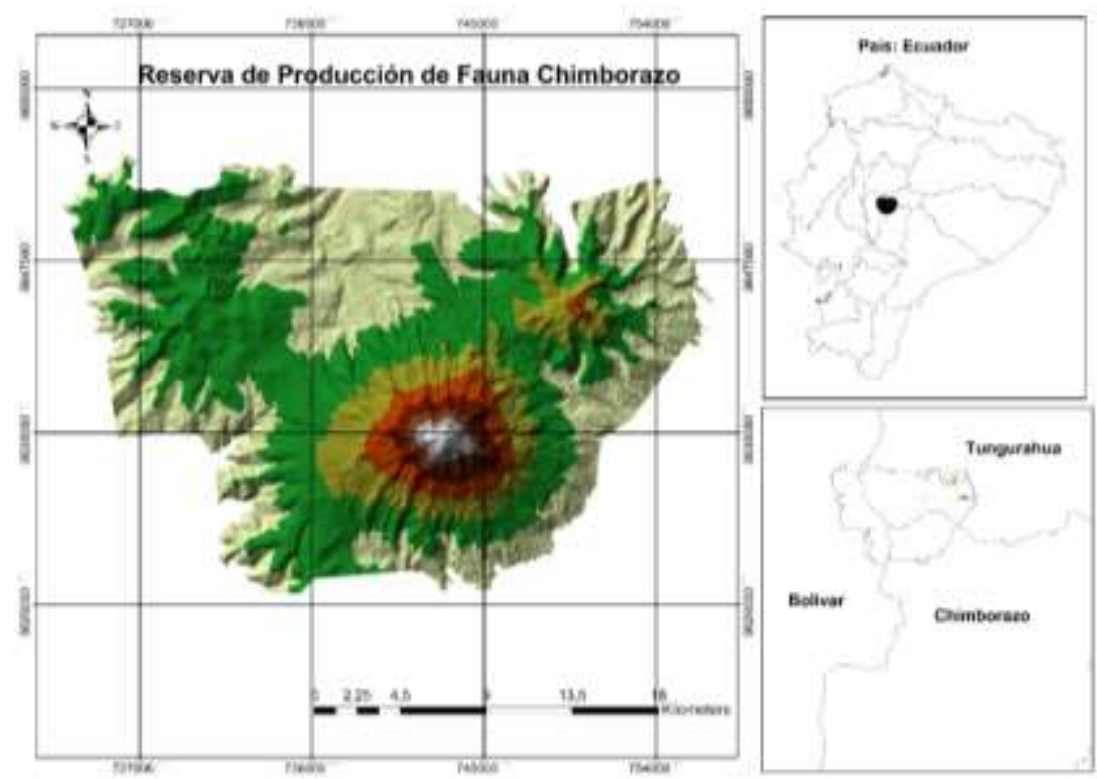

Nota: Se ubica en las provincias de Chimborazo, Tungurahua y Bolívar

Fuente: Elaboración propia (2021) 


\section{Hábitat de la vicuña}

El hábitat natural de la vicuña está conformado por el paisaje altoandino entre los 3.000 y 4.800 m.s.n.m., la vicuña es un animal que se encuentra en estado silvestre, gregario y territorial (Lichtenstein et al., 2002; Torres, 1983; Vilá B. et al., 2010). Un individuo requiere entre 3 y 5 hectáreas al año, abarcando un mínimo de 30\% de pastos de alta calidad principalmente en épocas húmedas (González, 2020; Korswagen Eguren, 2016; Lichtenstein et al., 2002; Vilá B. et al., 2010), por otro lado, en la época seca los individuos incluyen en sus dietas mayores proporciones de arbustos, tales como Junellia seriphioides y Artemisia copa, y otras especies de pastos menos nutritivas como Festuca sp. y Deyeuxia sp (González, 2020). En esencia los pastos naturales que forman el hábitat principal de la vicuña son gramíneas de los géneros Festuca, Stipa, Calamagrostis, y Poa entre otros (Korswagen Eguren, 2016; Lichtenstein et al., 2002), por tamto prefieren sitios con alta cobertura vegetal y dominio de gramíneas (Benitez et al., 2009).

La especie, si bien es generalista, es selectiva con la calidad de pastos que ingiere (Korswagen Eguren, 2016), su desarrollo esta dado por factores como la disponibilidad y calidad nutricional del forraje, cercanía a las fuentes de agua y otros factores ambientales como los disturbios antrópicos (Rojo \& Yanira, 2012). La zona noroeste de la RPFCH alberga camélidos, concretamente las zonas del Sinche y Mecha Huasca, esto se debe a que la liberación de los individuos en el proceso de reintroducción de produjo en ambas zonas pues cumplen con los requerimientos de la especie. Al referirnos a formaciones vegetales el herbazal ultrahúmedo subnival del páramo aglutina aproximadamente $23 \%$ del área total del área protegida, y el $42 \%$ de población de vicuña.

La vicuña comparte hábitat con mamíferos como el venado de cola blanca, y mamíferos pequeños ( Bayancela Delgado \& Cajas Bermeo, 2020), tiene como depredador natural al zorro (González, 2020; Rodríguez González \& Morales De La Nuez, 2017). Se ha observado que actividades como la cacería y atropellamientos son las principales causas de mortandad.

\section{Metodologia.}

El diseño de la investigación fue no experimental, de tipo de campo dentro de los límites de la Reserva de Producción de Fauna Chimborazo y de nivel evaluativo de las contribuciones de las variables para establecer la idoneidad del hábitat de la vicuña que propone lineamientos y criterios ambientales, topográficos y antrópicas para un manejo sostenible de la especie vicugna vicugna.

La investigación contempló tres etapas, la primera consistió en la caracterización del área de estudio, la selección de los elementos de la investigación, el registro de presencia de Vicugna vicugna (Tirira, 2015), y la preparación de los datos, esta etapa se sustentó en búsqueda bibliográfica y reunión con actores locales y funcionarios del Ministerio del Ambiente Agua y Transición ecológica (MAATE), así como también consultas a expertos, para complementar esta etapa fueron necesarias diversas salidas de campo. 
Los datos correspondientes a ocurrencias de Vicugna Vicugna (Tirira, 2015), fueron compilados por investigadores del proyecto "Determinación de la capacidad de carga de la Vicuña en la Reserva de Producción de Fauna Chimborazo", desde febrero 2020 a julio 2021 para lo cual se ejecutaron varios recorridos en campo recopilando datos de registros de presencia de vicuñas mediante técnicas de observación directa (Bayancela Delgado \& Cajas Bermeo, 2020; González, 2020), los recorridos abarcaron tramos de longitud comprendidos entre 1 a 3 kilómetros, incluyeron distintos tipos de vegetación y usos de suelo. Los puntos fueron georreferenciados en campo mediante una unidad GPS (Global Positioning System), se registraron 713 ocurrencias en total. Finalmente, la información recolectada (coordenadas) fue procesada en el software ArcGIS 10.5 (Akter et al., 2020), usando el Sistema de Referencia Universal Transverse Mercator (UTM), World Geodetic System (WGS 1984), zona 17 (Gavilanes Montoya et al., 2021).

La segunda etapa consistió en la preparación de las capas de las variables para esto se prepararon las capas ráster ligadas a variables ambientales y de factores antrópicos.

Las coberturas bioclimáticas se obtuvieron del data set WorldClim (Fick \& Hijmans, 2017; Plasencia-Vázquez et al., 2014; Vistin Guamantaqui et al., 2021), con una resolución de 30 segundos (Varela et al., 2015), las variables consideradas para el estudio fueron: temperatura, precipitación y velocidad del viento.

Estas conforman un conjunto de factores abióticos que las variables topográficas (Plasencia-Vázquez et al., 2014), fueron incorporadas mediante la variable modelo de elevación generado a partir de las cartas topográficas N-IV (C1, C2 y C3, y usando las herramientas CREATE TIN (3D Analyst) y TIN TO RASTER (3D Analyst) se obtuvo la variables en formato ráster.

Para incorporar la situación actual de los componentes bióticos y abióticos se incluyó las variables NDVI (Índice de Vegetación de Diferencia Normalizada) y NDWI (índice diferencial de agua normalizado) determinados mediante la combinación de bandas de la imagen satelital LANDSAT 8, con baja nubosidad y representativa de la variabilidad estacional a lo largo del año 2017, para el NDVI se usaron las bandas Infrarrojo cercano (NIR) y rojo y para el NDWI se usaron las bandas Infrarrojo cercano (NIR) y verde, esto se lo realizo en el Arc Map 10.4.1 utilizando la herramienta RASTER CALCULATOR (Vázquez, 2017). La perturbación humana fue añadida mediante la variable cobertura y uso de la tierra del Ecuador continental del año 2018, el primer nivel de la leyenda corresponde a las 6 clases definidas por el IPCC (Grupo Intergubernamental de Expertos sobre el Cambio Climático) (IPCC, 2019).

Se añadió la conectividad de los hábitats mediante la variable ecosistemas que representa la caracterización, localización y distribución de los ecosistemas para el año 2012, a escala 1: 100000 a partir de modelamientos biofísicos, interpretación de imágenes satelitales (2010-2012) y validación en campo. Finalmente se homologó los raster para el tamaño de celda (30 x 30) con un número de columnas de 1138 y 768 filas y con la proyección WGS 198417 SUR (Gavilanes Montoya et al., 2021), y se transformaron en formato ASCII según requerimientos del software Maxent (Cartaya Ríos et al., 2016). 
En la tercera etapa se corrieron varios modelamientos del hábitat potencial de la vicuña con Maxent (versión 3.4.1) hasta seleccionar las variables ambientales más relevantes para la especie y el ámbito de estudio (Ortega-Huerta \& Peterson, 2008)Los modelos se evaluaron comparando los resultados, indicadores sobre su performance, la literatura y el conocimiento de campo sobre la vicuña y su hábitat. Adicionalmente se efectuó el cruce e interpretación de resultados, así como la elaboración de mapas y productos finales. Esta se apoyó en el análisis SIG de los diversos resultados y su discusión. Se examinaron las relaciones espaciales entre el uso del suelo y el hábitat actual y potencial de la vicuña. Cabe indicar que seleccionamos al azar el $90 \%$ de los puntos de distribución de especies para construir el modelo, y el 10\% restante de los puntos de distribución de especies para probar el modelo. Las pruebas de Jackknife se utilizaron para analizar la tasa de contribución y la importancia de las variables, y la precisión del modelo puede considerarse excelente si el valor de AUC( área bajo la curva) está entre 0.9 y 1, bueno si el valor de AUC está entre 0.8 y 0.9, y determinarse como falló si el valor de AUC está entre 0.5 y 0.6 (Swets, 1988).

Los mapas de idoneidad se calcularon utilizando la salida logística de Maxent, que trabaja bajo el supuesto de que se puede asignar un valor conocido de probabilidad de observación a los pixeles llamados "típicos", básicamente es una aproximación a la verdadera probabilidad de presencia, si el índice lógico de idoneidad del hábitat es "0" significa que bajo y "1" es alto (Nieto Masot et al., 2016).

Dividimos los mapas de idoneidad del hábitat en cuatro niveles de acuerdo al siguiente criterio:

Tabla 1

\begin{tabular}{ll}
\multicolumn{2}{c}{ Niveles de idoneidad de hábitat } \\
\hline VALOR & SIGNIFICADO \\
\hline $\mathbf{0 , 6}$ a $\mathbf{1}$ & Altamente adecuado \\
\hline $\mathbf{0 , 4}$ a $\mathbf{0 , 6}$ & Moderadamente adecuado \\
\hline $\mathbf{0 , 2}$ a $\mathbf{0 , 4}$ & moderadamente bajo \\
\hline $\mathbf{0}$ a $\mathbf{0 , 2}$ & no adecuado
\end{tabular}

Fuente: elaboración propia (2021).

\section{Resultados}

\section{Rendimiento del modelo}

Los métodos de modelización ofrecen resultados normalmente muy diferentes entre sí, por lo que la selección del método que será utilizado en la interpretación es muy importante en ocasiones los resultados pueden resultar contradictorios (Austin, 2002), en este caso el aumento de entrenamiento regularizado es 1.272, el AUC de entrenamiento es 0.960 , el aumento de entrenamiento no regularizado es 1.811. El AUC de prueba es 
0.776, la desviación estándar es 0.016, lo que indica que el resultado de la predicción está cerca o por encima del nivel excelente. Por lo tanto, el modelo fue altamente informativo y podría usarse para investigaciones posteriores.

Figura 2.

Curva operacional ROC y el AUC.

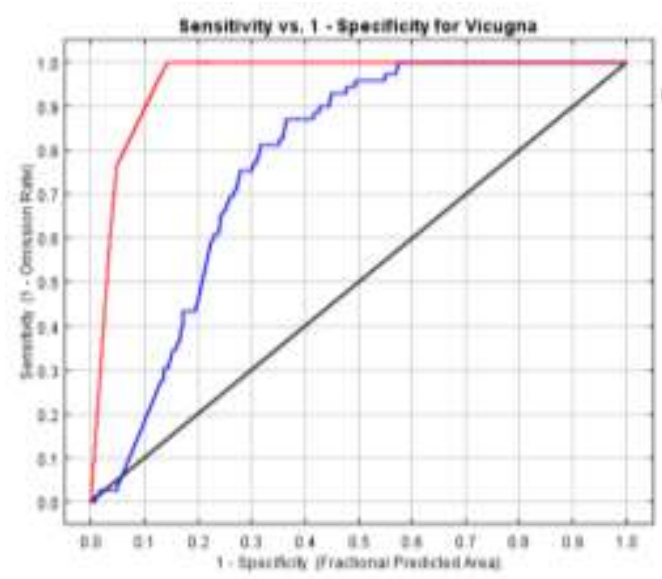

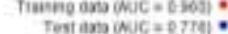

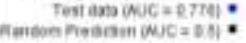

Nota: Análisis AUC de los modelos de distribución actual y potencias de la especie vicugna vicugna en la RPFCH

Fuente: Elaboración propia (2021).

Importancia ambiental variable

Tabla 2.

Contribución de las variables

Variable

\section{Porcentaje de contribución Importancia de permutación}

\begin{tabular}{lll}
\hline NDVI & 31.6 & 0 \\
\hline Modelo de elevación & 21.6 & 0,3 \\
\hline Precipitación & 19.2 & 56.1 \\
\hline NDWI & 8.2 & 0 \\
\hline Viento & 7.2 & 22.6 \\
\hline Uso de suelo & 6.3 & 4.4 \\
\hline Temperatura & 4.4 & 12.8 \\
\hline Ecosistemas & 1.2 & 3.8
\end{tabular}

Fuente: Elaboración propia (2021).

Las variables empleadas y su relevancia se muestran en Maxent mediante el test de Jacknife como un análisis de la contribución de cada variable en la determinación del hábitat modelado (Korswagen Eguren, 2016; Merow et al., 2013), bootstrap para el remuestreo, de esta manera se disminuye el sesgo de la muestra; y el área bajo la curva 
(AUC) para evaluar la apttud del modelo y rendimiento (Cartaya Ríos et al., 2016), en nuestro estudio las variables que contribuyeron en mayor medida a la idoneidad del hábitat de Vicugna vicugna fueron: NDVI modelo de elevación y precipitación, la tasa de contribución total de estos factores alcanzó el 72,4\%, siendo la primera la más relevante $(31,6 \%)$.

\section{Análisis de variable ambiental y física}

El rango óptimo para NDVI fue de $-0,32$ a 0,47 presente en zonas con poca vegetación o arenales y zonas con vegetación de pajonales concordando con lo manifestado por Caranqui \& Pino (2015) en cuyo estudio Vicugna vicugna prefiere Elymus cordilleranus, y Calamagrostis intermedia especies características de los ecosistemas Herbazal ultrahúmedo subnival del páramo y Herbazal húmedo montano alto superior del Páramo(Ministerio del ambiente del Ecuador, 2013).

El rango óptimo del modelo de elevación digital esta entre 3950 y 4960 metros sobre el nivel del mar (msnm) corresponde a las áreas que circundan los nevados Chimborazo y Carihuayrazo concretamente. En cuanto a la variable precipitación total el rango óptimo va de 508 a $680 \mathrm{~mm}$ lo que indica que el hábitat idóneo de la especie está ligada a zonas con menor pluviosidad.

\section{Mapa de idoneidad del hábitat}

Figura 3.

Distribución espacial de la vicuña

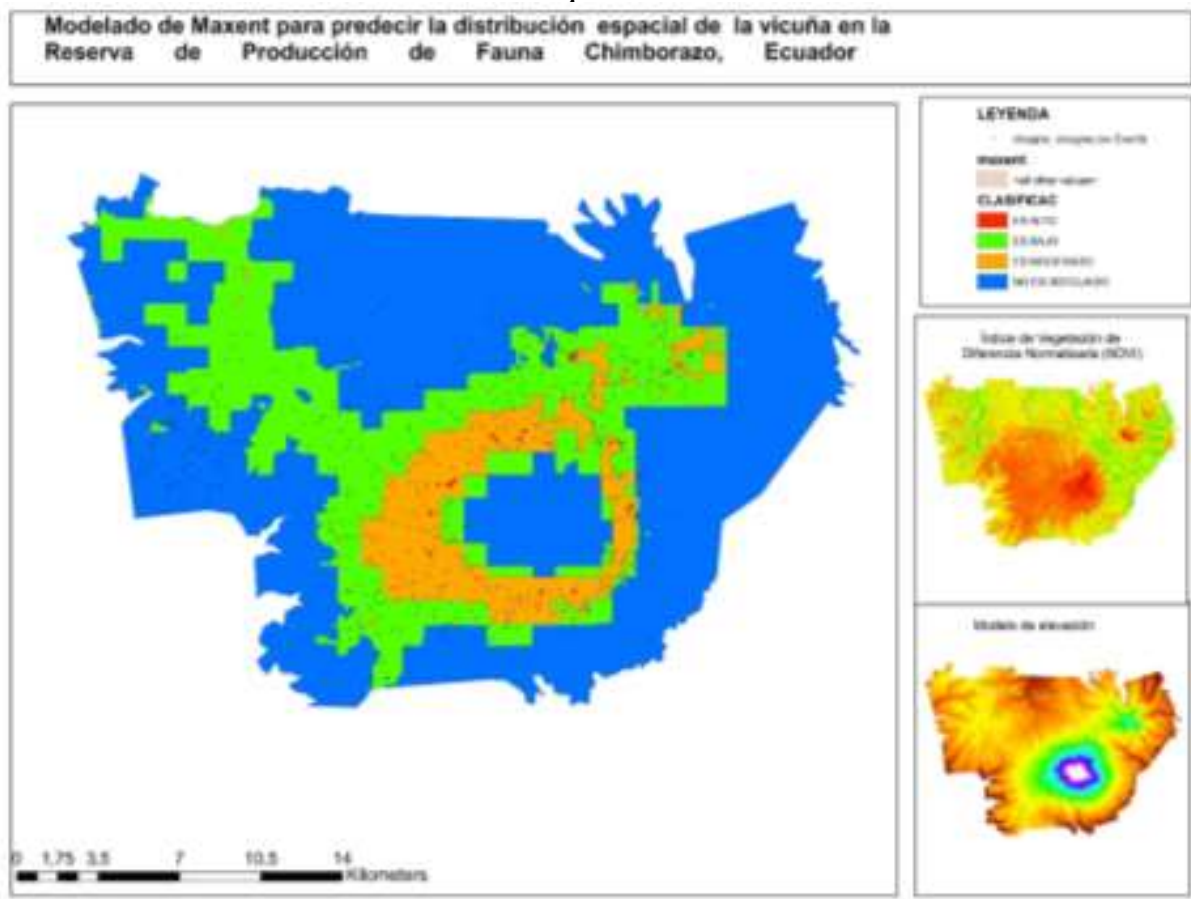

Nota: modelado maxent para predecir distribución espacial de la vicuña en la Reserva de Producción de Fauna Chimborazo.

Fuente: Elaboración propia (2021). 
De acuerdo con los mapas de distribución de idoneidad y la proporción de idoneidad de la vicuña, las áreas de coloración roja corresponden al hábitat altamente adecuado (54,18 has), mientras que la coloración naranja está ligada al hábitat moderadamente adecuado (5343,42 has) que representa el 10,1\% del área de estudio, el área definida como hábitat moderadamente bajo alcanza 13743,63 has representando 26,1\% del área en el mapa presenta una coloración verde, finalmente el área determinada como no adecuada corresponde a la mayor parte del AP ( 63,7 \%) se presenta en tonos azules indican baja probabilidad de condiciones ambientales para el desarrollo de la especie (Cartaya Ríos et al., 2016).

\section{Figura 4}

\section{Distribución idoneidad del hábitat de la vicuña}

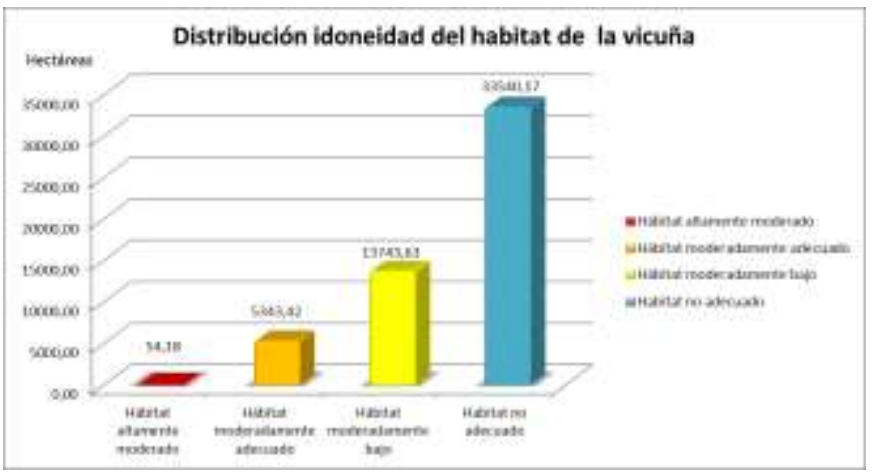

Nota: Niveles de clasificación de idoneidad del hábitat de la vicuña.

Fuente: Elaboración propia (2021).

Al realizar el análisis a nivel parroquial se determina que la zona con alta probabilidad de condiciones favorables (hábitat altamente moderado) para la Vicugna vicugna, se encuentra al sur de Pilahuín (71,1\%) perteneciente a la provincia de Tungurahua con gran proximidad a la base de los nevados Carihuyrazo y Chimborazo, entre tanto 28,9\% corresponden a San Andrés y San Juan situadas en la provincia de Chimborazo. Gran parte del hábitat moderadamente adecuado para la vicuña se ubica en las parroquias de Pilahuín, San Juan y San Andrés con un 41\%, 38,6 \% y 13,1 \% respectivamente, en tanto que parroquias como Guanujo, Salinas, Mocha y Quinchicoto presentan valores porcentuales inferiores a 5 .

En relación con el hábitat moderadamente bajo donde se registraron presencias de vicuñas en menor densidad, el mayor porcentaje $(45,4)$ se presenta en la zona noroeste de la parroquia de Pilahuín correspondiente a 6238,7 has, en tanto que Guanujo y Salinas localizadas en la provincia de Bolívar abarcan valores porcentuales entre 23,9 y 11,7 respectivamente, por otra parte, San Juan y San Andrés presentan estas zonas con hábitat moderadamente bajo en menor dimensión $(9,9 \%$ y $6,4 \%)$.

Considerando que la parroquia de Pilahuin posee la mayor cantidad de su territorio dentro de los límites de la Reserva de Producción de Fauna Chimborazo, contiene los cuatro 
niveles de idoneidad en el caso de las zonas definidas como hábitat no adecuado para las vicuñas abarca el 34,9\% ubicados al noreste y en las zonas bajas que poseen un rango altitudinal que va de 3800 a 4000, le siguen las parroquias de Guanujo y San Andrés con valores porcentuales entre $18,6 \% 16,2 \%$ respectivamente mientras que parroquias como Mocha, San Juan Salinas Quinchicoto, Santa Rosa, Juan Benigno Vela y Simiatug presentan valores porcentuales menor a 10 .

A manera de resumen las zonas que presentan las condiciones ambientales más favorables para la especie se encuentran en la zona central de la reserva de producción de fauna Chimborazo alrededor de los nevados Chimborazo y Carihuayrazo.

\section{Discusión}

Maxent varía en respuesta a diferentes configuraciones para resaltar la necesidad de tomar decisiones de modelado motivadas biológicamente (Merow et al., 2013), el rendimiento está influenciado por un número moderado de parámetros (Phillips \& Dudík, 2008), la configuración predeterminada ajustada a los datos de solo presencia logra un rendimiento que es casi tan bueno como si se hubiera ajustado a los datos de evaluación en sí (Phillips et al., 2006; Phillips \& Dudík, 2008).

El enfoque de validación jackknife permite la evaluación de la capacidad predictiva de los modelos (Merow et al., 2013), el estadístico AUC, no bajo de 0,960 lo que indica una buena discriminación entre presencia/ausencia tal como los plantean los autores (Macías Barrero, 2016). De acuerdo con los valores de AUC, las variables abióticas que funcionaron como mejores predictores de la distribución de la Vicugna vicugna fueron; el índice de vegetación de diferencia normalizada (NDVI), el modelo de elevación y la precipitación fueron los principales factores que afectaron la idoneidad de su hábitat, con una tasa de contribución total de estos tres factores que alcanzó el 72,4\%, lo que concuerda con los estudios de Korswagen (2016) que establece como variables más determinantes para la idoneidad del hábitat de la vicuña a la oscilación diurna de la temperatura, precipitación de los meses más lluviosos y precipitación de los meses más cálidos (verano); índices de vegetación VI y SRSR luego de la estación seca (agosto), así como la estacionalidad en la precipitación y la precipitación en los meses más fríos, otros índices de vegetación para agosto y el modelo digital de elevación.

Los autores (Cartaya Ríos et al., 2016) trabajan con variables bioclimáticas y define como variables más relevantes las asociadas a precipitaciones del trimestre más frío, del más cálido y del más seco, seguido de la altitud. Por otro lado de acuerdo al mismo autor, la elevación se asocia con varios parámetros que pueden influir la distribución de la especie porque está relacionada con la presencia de ecosistemas óptimos para la especie, en concordancia con lo manifestado por el autor (González, 2020), ya que este factor muestra la incidencia de la altitud en la alimentación de vicuñas, lo expuesto coincide con el presente estudio de tal forma que los índices espectrales para vegetación se convierten en una herramienta útil para el estudio del hábitat de la vicuña, además la precipitación, el modelo de elevación y la atura son factores restrictivos para predecir la distribución espacial. 
Según Thapa et al., (2018) "un aumento de los impactos antropogénicos acelera el declive de especies" criterio que concuerda con nuestro estudio puesto que el hábitat potencial de la especies se localiza en sectores alejados de zonas pobladas ya que las actividades humanas como agricultura tradicional, ganadería afectan la distribución de vicugna vicugna (Ibarra-Montoya et al., 2014).

\section{Conclusiones}

- El factor que más contribuye a la idoneidad del hábitat de la Vicugna vicugna es el Índice de vegetación de diferencia normalizada, puesto que este índice espectral detecta zonas con densidad de biomasa baja, pero con calidad nutricional del forraje lo que restringe el hábitat de la vicuña, por otro lado el modelo de elevación es un factor determinante para definir el habitat de la vicuña ya que es una especie con rangos restringidos de altura, en el estudio se aprecia el rango optimo se ubica entre $3950 \mathrm{msnm}$ a 4960 metros sobre el nivel del mar y finalmente el factor que contribuyente precipitación delimita las zonas donde se desarrolla la especie, puesto que las zonas ubicadas al Noreste y Suroeste del área protegida presenta mayor pluviosidad y así mismo menor número de áreas definidas como hábitat altamente moderado.

- La mayor parte del territorio $(63,7 \%)$ de la reserva Chimborazo se considerada como no adecuada, es decir no reúne los factores óptimos para ser definida como hábitat de la vicuña, las zonas se ubican por debajo de los 3950 metros sobre el nivel del mar, son áreas con presencia de una alta y media densidad de vegetación arbórea y herbácea, con abundantes lluvias y presencia de asentamientos humanos (42 comunidades) que realizan prácticas agrícolas y pecuarias a lo largo de todo el perímetro del área protegida. El área definida como hábitat moderadamente bajo tiene 13743,63 hectáreas que representa el 26,1\% del área protegida, son áreas potenciales de ser ocupadas por poblaciones de vicuñas en los próximos años ya que reúnen ciertos factores y rangos de distribución, se aprecian áreas importantes de distribución espacial potencial en las parroquia de Guanujo y Salinas en la provincia de Bolívar que corresponde a paramos con vegetación escasa y baja y existe poca intervención antrópica, que se dedica principalmente al pastoreo de ovejas.

- El 10,1\% del territorio corresponde al hábitat moderadamente adecuado que indica que las zonas tienen la mayoría de factores y cumplen con los rangos de distribución se ubican principalmente alrededor del nevado Chimborazo y existen pequeños parches alrededor del nevado Carihuayrazo, caracterizados por ser zonas altas sin presencia de intervención antrópica ubicada sobre la frontera agrícola, en estas zonas se realizan monitoreos de la biodiversidad y control y vigilancia por parte de los guardaparques del AP. Finalmente solo el 0,1\% del área total de la reserva Chimborazo (52683 hectáreas) son altamente adecuadas y se convierten en el hábitat óptimo de la vicuña ya que cumplen con todos los factores y rangos, se puede concebir como el espacio que reúne las condiciones y características físicas y biológicas favorables para la supervivencia y reproducción 
de la vicugna vicugna, son parches localizadas en las parroquias de Pilahuín, San Andrés y San Juan, mientras que el modelo no detecta áreas óptimas en la provincia de Bolívar.

\section{Referencias bibliográficas}

Akter, A., Uddin, A. M. H., Wahid, K. Ben, \& Ahmed, S. (2020). Predicting groundwater recharge potential zones using geospatial technique. Sustainable Water Resources Management, 6(2), 1-13. https://doi.org/10.1007/s40899-020-00384-w

Albán-Guaman, M. (2009). Estudio del hábitat y costumbres de las vicuñas en la Reserva de Producción de Fauna Chimborazo (Issue 57) [Tesis de pregado, Escuela Superior Politécnica de

Chimborazo]. http://dspace.espoch.edu.ec/handle/123456789/44/browse?type=author\&order=AS $\mathrm{C} \& \mathrm{rpp}=20 \&$ value $=\mathrm{Alban}+\mathrm{Guaman} \% 2 \mathrm{C}+\mathrm{Marco}+$ Fernando

Ati-Cutiupala, G. M., Muñoz-Jácome, E. A., Vistín-Guamantaqui, D. A., \& Cushquicullma-Colcha, D. F. (2021). Análisis de las actividades económicas de tres comunidades altoandinas y su relación con el cambio de uso de suelo y cobertura vegetal en la Reserva de Producción de Fauna Chimborazo. Polo de Conocimiento, 6(8), 552-576. https://doi.org/10.23857/pc.v6i8.2956

Austin, M. P. (2002). Spatial prediction of species distribution: An interface between ecological theory and statistical modelling. Ecological Modelling, 157(2-3), 101118. https://doi.org/10.1016/S0304-3800(02)00205-3

Bayancela Delgado, S. B., \& Cajas Bermeo, C. A. (2020). Vertebrados terrestres del ecosistema Herbazal húmedo subnival del páramo en la Reserva de Producción de Fauna Chimborazo. ConcienciaDigital, 3(3), 127-140. https://doi.org/10.33262/concienciadigital.v3i3.1285

Benitez, V., Borgnia, M., \& Cassini, M. H. (2009). Ecología nutricional de la vicuña (Vicugna vicugna): Un caso de estudio en la Reserva Laguna Blanca, Catamarca. Investigación, Conservación y Manejo de Vicuñas, July, 17. http://link.springer.com/10.1007/978-0-387-09476-2

Caranqui, J., \& Pino, M. (2015). Especies Alimenticias De La Vicuña En La Reserva De Producción Faunística Chimborazo, Ecuador. Artículos Forestal, March, 6.

Cartaya Ríos, S. J., Anchundia Betancuort, C. E., \& Mantuano Eduarte, R. A. (2016). DISTRIBUCIÓN GEOGRÁFICA POTENCIAL DE LA ESPECIE CUNICULUS PACA (Guanta) EN EL OCCIDENTE DE ECUADOR. La Granja, 24(2), 134-149. https://doi.org/10.17163/lgr.n24.2016.09

Fick, S. E., \& Hijmans, R. J. (2017). WorldClim 2: new 1-km spatial resolution climate surfaces for global land areas. International Journal of Climatology, 37(12), 43024315. https://doi.org/10.1002/joc.5086 
García-Huamaní, R. (2020). Comercialización de la fibra de vicuña en La Reserva Nacional De Pampa Galeras Bárbara D’Achille. Ciencia Unemi, 13(33), 18-31. https://doi.org/10.29076/issn.2528-7737vol13iss33.2020pp18-31p

Gavilanes Montoya, A. V., Esparza Parra, J. F., Chávez Velásquez, C. R., Tito Guanuche, P. E., Parra Vintimilla, G. M., Mestanza-Ramón, C., \& Vizuete, D. D. C. (2021). A Nature Tourism Route through GIS to Improve the Visibility of the Natural Resources of the Altar Volcano, Sangay National Park, Ecuador. Land, 10(8), 884. https://doi.org/10.3390/land10080884

González, B. A. (2020). La vicuña austral (Issue Enero). Universidad de Chile.

Hernández, O. G., Artigas, R. C., González, J. M. S., \& García, L. V. (2018). Predictive modeling in Biogeography: Applying ecological niche modelling in Physical Geography. Boletin de La Asociacion de Geografos Espanoles, 2018(78), 88-126. https://doi.org/10.21138/bage.2395

Herrador-Valencia, D., \& Paredes, M. (2016). Cambio climático y agricultura de pequeña escala en los Andes ecuatorianos: un estudio sobre percepciones locales y estrategias de adaptación. Journal of Latin American Geography, 15(2), 101-121. https://doi.org/10.1353/lag.2016.0021

Hofstede, R. (2004). Health state of Páramos : an effort to correlate science and. Lyonia, 6(December), 61-73. http://origin.portalces.org/sites/default/files/references/039_Hofstede. 2004. Lyonia.pdf

Hofstede, R., Vasconez, S., \& Cerra, M. (2015). VIVIR EN LOS PARAMOS.Percepciones, vulnerabilidades, capacidades y gobernanza ante el cambio climático (R. Hofstede, S. Vasconez, \& M. Cerra (eds.)). Unión Internacional para la Conservación de la Naturaleza y de los Recursos Naturales. UICN. http://www.uicn.org/sur

Ibarra-Montoya, J., Rangel-Peraza, G., González-Farias, F., Enrique, M.-M., \& MaciasCuellar, H. (2014). Uso del modelado de nicho ecológico como una herramienta para predecir la distribución potencial de Microcystis sp (cianobacteria) en la Presa Hidroeléctrica de Aguamilpa, Nayarit, México. Revista Ambiente e Agua, 9(3), 445458. https://doi.org/10.4136/1980-993X

IPCC. (2019). El Informe Especial del IPCC sobre Cambio Climático y la Tierra ¿ Qué significa para América Latina? Ipcc, 34. https://bit.ly/2Yp0UKD

Jara, C., Delegido, J., Ayala, J., Lozano, P., Armas, A., \& Flores, V. (2019). Study of wetlands in the ecuadorian andes through the comparison of landsat- 8 and sentinel2 images. Revista de Teledeteccion, 2019(53), 45-57. https://doi.org/10.4995/raet.2019.11715 
Josse, C., Navarro, G., Comer, P., Evans, R., Langendoen, F., Fellows, M., Kittel, G., Menard, S., Pyne, M., Reid, M., Schulz, K., Snow, K., \& Teague, J. (2003). Ecological Systems of Latin America and the Caribbean. A working Classification of Terrestrial Siystems. In fhttps://pdfs.semanticscholar.org/f637/8807838f8f8cdba6d3dcf5ec894cc65e8c7e.p df?_ga=2.91414170.1099870656.1569594671-633467499.1569594671

Korswagen Eguren, S. (2016). Análisis espacial del hábitat de la vicuña en la comunidad campesina de Tanta, en la Reserva Paisajística Nor Yauyos Cochas Spatial analysis of vicugna's habitat in a Peasant Community in Nor Yauyos Cochas Landscape Reserve. Espacio y Desarrollo, 128(28), 103-128. https://doi.org/10.18800/espacioydesarrollo.201601.005

Lichtenstein, G., Oribe, F., Grieg-gran, M., \& Mazzucchelli, S. (2002). Manejo Comunitario de Vicuñas en Perú: Estudio de caso del manejo comunitario de vida silvestre. $2,82$.

Macías Barrero, J. M. (2016). Distribución actual y potencial de especies leñosas alimenticias en África para la formulación de estrategias de conservación. [Tesis de maestría,Universidad Complutense de Madrid]. https://eprints.ucm.es/id/eprint/40046/1/TFM.pdf

MAE. (2014). MAE (Ministerio del Ambiente del Ecuador) Actualización del plan de manejo de la Reserva de producción de Fauna Chimborazo, 1-297.

Merow, C., Smith, M. J., \& Silander, J. A. (2013). A practical guide to MaxEnt for modeling species' distributions: What it does, and why inputs and settings matter. Ecography, 36(10), 1058-1069. https://doi.org/10.1111/j.1600-0587.2013.07872.x

Ministerio del ambiente del Ecuador. (2013). Sistema de clasificación de los Ecosistemas del Ecuador Continental (R. Galeas, J. Guevara, B. Medina, M. A. Chinchero, \& H. Ximena (eds.)). http://app.sni.gob.ec/sni-link/sni/PDOT/NIVEL NACIONAL/MAE/ECOSISTEMAS/DOCUMENTOS/Sistema.pdf

Ministerio del Ambiente del Ecuador. (2002). PLAN GERENCIAL RESERVA DE PRODUCCIÓN FAUNISTICA CHIMBORAZO RESUMEN EJECUTIVO. http://suia.ambiente.gob.ec/documents/10179/242256/35+PLAN+DE+MANEJO+ CHIMBORAZO.pdf/d116d0db-aefc-477b-8188-f4a627af486d

Ministerio del Ambiente del Ecuador. (2014). Actualizacion del Plan de Manejo de la Reserva de Produccion de Fauna Chimborazo.

Nieto Masot, A., Navazo Arenas, G., \& Moreno Marcos, G. (2016). Tecnologías de la información geográfica en el análisis espacial : aplicaciones en los sectores público, empresarial y universitario. In Junta de extremadura. https://dialnet.unirioja.es/servlet/articulo?codigo=5776243 
Ortega-Huerta, M. A., \& Peterson, A. T. (2008). Modeling ecological niches and predicting geographic distributions: A test of six presence-only methods. Revista Mexicana de Biodiversidad, 79(1), 205-216. https://doi.org/10.22201/ib.20078706e.2008.001.522

Phillips, S. B., Aneja, V. P., Kang, D., \& Arya, S. P. (2006). Modelling and analysis of the atmospheric nitrogen deposition in North Carolina. International Journal of Global Environmental Issues, 6(2-3), 231-252. https://doi.org/10.1016/j.ecolmodel.2005.03.026

Phillips, S. J., \& Dudík, M. (2008). Modeling of species distributions with Maxent: New extensions and a comprehensive evaluation. Ecography, 31(2), 161-175. https://doi.org/10.1111/j.0906-7590.2008.5203.x

Plasencia-Vázquez, A. H., Escalona-Segura, G., \& Esparza-Olguín, L. G. (2014). Modelación de la distribución geográfica potencial de dos especies de psitácidos neotropicales utilizando variables climáticas y topográficas. Acta Zoológica Mexicana (N.S.), 30(3), 471-490. https://doi.org/10.21829/azm.2014.30372

Pliscoff, P., \& Fuentes-Castillo, T. (2011). Modelación de la distribución de especies y ecosistemas en el tiempo y en el espacio: Una revisión de las nuevas herramientas y enfoques disponibles. Revista de Geografia Norte Grande, 2011(48), 61-79. https://doi.org/10.4067/s0718-34022011000100005

Podwojewski Pascal, Poulenard Jérôme, Zambrana Tania, \& Hofstede Robert. (2002). Efectos de pastoreo excesivo sobre la cubierta vegetal y las propiedades del suelo de cenizas volcánicas en el páramo de Llangahua y La Esperanza (Tungurahua, Ecuador). Uso y Manejo Del Suelo. https://doi.org/10.1111 / j.14752743.2002.tb00049.x

Rodríguez González, N. F., \& Morales De La Nuez, A. (2017). La vicuña ecuatoriana y su entorno (Ministerio del Ambiente del Ecuador (ed.); Primera ed). junio 2017. http://maetransparente.ambiente.gob.ec/documentacion/Biodiversidad/LA_VICUÑ A_ECUATORIANA.pdf

Rojo, V., \& Yanira, B. (2012). Uso de habitad por vicuñas en un sistema agropastoril en suripujio,Jujuy. Mastozoología Neotropical, 127-138.

Romero, F., Muñoz, E., Arguello, C., Zurita, M., \& Roman, D. (2018). Hacia un manejo adaptativo de la Reserva de Producción de Fauna Chimborazo y su zona de amortiguamiento. www.giz.de

Sierra, R. (1999). Propuesta Preliminar de un Sistema de Clasificación de Vegetación para el Ecuador Continental.Proyecto INEFAN/GEF-BIRG Ecociencia. January 1999, 194. https://doi.org/10.13140/2.1.4520.9287

Swets, J. A. (1988). Measuring the Accuracy of Diagnostic Systems Author(s): John A. 
Swets Source: Science, 240(4857), 1285-1293.

Thapa, A., Wu, R., Hu, Y., Nie, Y., Singh, P. B., Khatiwada, J. R., Yan, L., Gu, X., \& Wei, F. (2018). Predicting the potential distribution of the endangered red panda across its entire range using MaxEnt modeling. Ecology and Evolution, 8(21), 10542-10554. https://doi.org/10.1002/ece3.4526

Tirira, D. G. (2015). Lista Actualizada De Especies. Mamiferos Del Ecuador, 1-49.

Torres, H. (1983). Distribución y Conservación de la vicuña (Vicugna vicugna) (pp. 528). https://portals.iucn.org/library/sites/library/files/documents/1983-001.pdf

Varea, A. (2004). Redalyc.Iniciativas para conservar la biodiversidad. Universitas, Revista de Ciencias Sociales y Humanas, IV, 7-43.

Varela, S., Terribilie, L., \& OLivieira, G. (2015). ecoClimate vs. Worldclim: variables climáticas SIG para trabajar en biogeografía. Ecosistemas, 24(3), 88-92. https://doi.org/10.7818/re.2014.24-3.00

Vásconez, P., Medina, G., \& Hofstede, R. (2001). Los Páramos del Ecuador. In Botánica económica de los Andes Centrales (Vol. 2006).

Vázquez, R. (2017). Uso De Sistemas De Información Geográfica Libres Para La Protección Del Medio Ambiente. Caso De Estudio: Manipulación De Mapas Ráster Con Datos Climáticos. Universidad y Sociedad, 10(2), 158-164. http://scielo.sld.cu/pdf/rus/v10n2/2218-3620-rus-10-02-158.pdf

Vilá B., Wawrzyk A., \& Arzamendia Y. (2010). El manejo de vicuñas silvestres (Vicugna vicugna) en Jujuy (Argentina): Un análisis de la experiencia del proyecto MACS, en Cieneguillas. R. Revista Latinoamericana de Conservación. 1 (1), 1(1), 38-52. http://lajoc.procat-conservation.org/ojs/index.php/procat/index

Vistin Guamantaqui, D. A., Muñoz Jacome, E. A., \& Ati Cutiupala, G. M. (2021). ESCENARIO DE CAMBIO CLIMÁTICO DEL BOSQUE SIEMPREVERDE DEL PÁRAMO RESERVA DE PRODUCCIÓN DE FAUNA CHIMBORAZOECUADOR. Tropical and Subtropical Agroecosystems, 24, 1-9. https://www.redalyc.org/journal/4760/476052525002/html/

Vistin Guamantaqui, D. A., Muñoz Jácome, E. A., \& Ati Cutiupala, G. M. A. C. (2020). Monitoreo del Herbazal del páramo una estrategia de medición del cambio climático en la Reserva de Producción de Fauna Chimborazo. Ciencia Digital, 4(2), 32-47. https://doi.org/10.33262/cienciadigital.v4i2.1195

\section{Ciencia Ligital}




\section{PARA CITAR EL ARTÍCULO INDEXADO.}

Vaca Cárdenas, M. L., Vaca Barahona, B. E., Cushquicullma Colcha, D. F., \& Ati Cutiupala, G. M. (2021). Modelado de Maxent, predicción de la distribución espacial de la vicuña en Ecuador. AlfaPublicaciones, 3(3.1), 406-424. https://doi.org/10.33262/ap.v3i3.1.109

El artículo que se publica es de exclusiva responsabilidad de los autores y no necesariamente reflejan el pensamiento de la Revista Alfa Publicaciones.

\section{LCiencia}

El artículo queda en propiedad de la revista y, por tanto, su publicación parcial y/o total en otro medio tiene que ser autorizado por el director de la Revista Alfa Publicaciones.
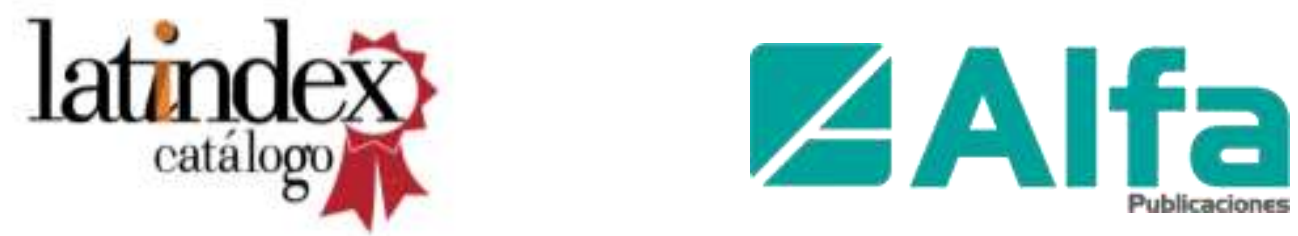